\title{
Should penalized least squares regression be interpreted as Maximum A Posteriori estimation?
}

\author{
Rémi Gribonval
}

\begin{abstract}
Penalized least squares regression is often used for signal denoising and inverse problems, and is commonly interpreted in a Bayesian framework as a Maximum A Posteriori (MAP) estimator, the penalty function being the negative logarithm of the prior. For example, the widely used quadratic program (with an $\ell^{1}$ penalty) associated to the LASSO / Basis Pursuit Denoising is very often considered as MAP estimation under a Laplacian prior in the context of additive white Gaussian noise (AWGN) reduction. This paper highlights the fact that, while this is one possible Bayesian interpretation, there can be other equally acceptable Bayesian interpretations. Therefore, solving a penalized least squares regression problem with penalty $\phi(x)$ need not be interpreted as assuming a prior $C \cdot \exp (-\phi(x))$ and using the MAP estimator. In particular, it is shown that for any prior $P_{X}$, the minimum mean square error (MMSE) estimator is the solution of a penalized least square problem with some penalty $\phi(x)$, which can be interpreted as the MAP estimator with the prior $C \cdot \exp (-\phi(x))$. Vice-versa, for certain penalties $\phi(x)$, the solution of the penalized least squares problem is indeed the MMSE estimator, with a certain prior $P_{X}$. In general $d P_{X}(x) \neq C \cdot \exp (-\phi(x)) d x$.
\end{abstract}

\section{INTRODUCTION}

Consider the problem of estimating an unknown signal $x \in \mathbb{R}^{n}$ from a noisy observation $y=x+b$, also known as denoising. Given an arbitrary noisy observation $y$ the goal is to estimate the noiseless signal $x$ : in practice, designing a denoising scheme amounts to choosing a function $\psi: \mathbb{R}^{n} \rightarrow \mathbb{R}^{n}$ which provides estimates of the form $\hat{x}=\psi(y)$. However, unless one specifies further what is meant by "noise" and "signal", denoising is a completely ill-posed problem since any pair $x, b$ such that $y=x+b$ can be replaced by a pair $x^{\prime}, b^{\prime}$ where $x^{\prime}=x+z$, $b^{\prime}=b-z$. Practical denoising schemes hence have to rely on various types of prior information on $x$ and $b$ to design an appropriate denoising function $\psi$.

\section{A. Bayesian estimation}

A standard statistical approach to the denoising problem consists in assuming that $x$ and $b$ are drawn independently at random from known prior probability distributions $P_{X}$ and $P_{B}$. Under this model, given a cost function $\mathcal{C}(\hat{x}, x)$ that measures the quality of an estimator $\hat{x}$ in comparison to the true quantity to estimate $x$, the Bayes estimator is defined as an estimator $\psi$ with minimum expected cost:

$$
\arg \min _{\psi} \mathbb{E}\{\mathcal{C}(\psi(X+B), X)\} .
$$

For a quadratic cost function $\mathcal{C}(\hat{x}, x):=\|\hat{x}-x\|_{2}^{2}$ the Bayes estimator is the minimum mean square error (MMSE) estimator [5], also called conditional mean, posterior mean, or conditional expectation:

$$
\psi_{\mathrm{MMSE}}(y):=\mathbb{E}(X \mid Y=y) .
$$

Rémi Gribonval is with INRIA, Centre Inria Rennes - Bretagne Atlantique, Campus de Beaulieu, F-35042 Rennes Cedex, Rennes, France. Phone: +332998425 06. Fax: +3329984 71 71. Email: remi.gribonval@inria.fr.

Rémi Gribonval is a member of the METISS project-team at IRISA, Rennes, France. This work was supported in part by the European Union through the project SMALL (Sparse Models, Algorithms and Learning for Large-Scale data). The project SMALL acknowledges the financial support of the Future and Emerging Technologies (FET) programme within the Seventh Framework Programme for Research of the European Commission, under FET-Open grant number: 225913.

NB: This work has been submitted to the IEEE for possible publication. Copyright may be transferred without notice, after which this version may no longer be accessible. 
Even though this estimator is "optimal" in the above defined sense, its computation involves a high-dimensional integral and cannot generally be done explicitly. In practice, Monte-Carlo simulations can be used to approximate the integral.

Often more amenable to efficient numerical optimization is the popular Maximum A Posteriori (MAP) criterion, which is the Bayes estimator associated to the $0-1$ cost function $(\mathcal{C}(\hat{x}, x)=1$, when $\hat{x} \neq x ; \mathcal{C}(\hat{x}, x)=0$, when $\hat{x}=x)$. Exploiting Bayes rule and assuming that both the noise and the unknown noiseless signal have probability density functions (pdf), $p_{X}$ and $p_{B}(b)$, the MAP estimator reads:

$$
\begin{aligned}
\psi_{\mathrm{MAP}}(y) & :=\arg \max _{x \in \mathbb{R}^{n}} p(x \mid y)=\arg \max _{x \in \mathbb{R}^{n}} p(y \mid x) p(x) \\
& =\arg \min _{x \in \mathbb{R}^{n}}\left\{-\log p_{B}(y-x)-\log p_{X}(x)\right\} .
\end{aligned}
$$

For white Gaussian noise $b$ we have $p_{B}(b) \propto \exp \left(-\|b\|_{2}^{2} / 2\right)$, where $\|b\|_{2}^{2}=\sum_{i=1}^{n} b_{i}^{2}$ and the notation $f(x) \propto g(x)$ means $f(x)=C \cdot g(x)$ for all $x$, with $C \neq 0$ some constant independent of $x$. Hence the MAP estimator under the prior $p_{X}(x)$ can be expressed as

$$
\psi_{\mathrm{MAP}}(y)=\arg \min _{x \in \mathbb{R}^{n}} \frac{1}{2}\|y-x\|_{2}^{2}+\left[-\log p_{X}(x)\right] .
$$

\section{B. Regularization}

Optimization problems of the type (I.2) have also been often considered in signal processing without explicit reference to probabilities or priors, under the generic form

$$
\arg \min _{x \in \mathbb{R}^{n}} \frac{1}{2}\|y-x\|_{2}^{2}+\phi(x) .
$$

The deterministic objective is to achieve a tradeoff between the data-fidelity term $\|y-x\|_{2}^{2}$ and the penalty term $\phi(x)$, which promotes solutions with certain properties. In particular, when the function $\phi$ is non-smooth at the origin, such as $\phi(x)=\|x\|_{p}^{p}:=\sum_{i=1}^{n}\left|x_{i}\right|^{p}, 0<p \leq 1$, the optimum of the criterion (I.3) is known to have few nonzero entries. Regularization with such penalty functions is at the basis of shrinkage techniques for signal denoising (see e.g. [3] with $p=1$, or [6] with $0<p \leq 1$ ). More recently, these approaches have become a very popular means of promoting sparse solutions to under-determined or ill-conditioned linear inverse problems $y=\mathbf{A} x+b$, and are now a key tool for compressed sensing [4].

\section{Plurality of Bayesian interpretations of regularization}

Given the identity of the optimization problems (I.2) and (I.3) when $\phi(x)=\phi_{\text {MAP }}(x):=-\log p_{X}(x)$, the regularization problem (I.3) is often interpreted ${ }^{1}$ as "solving the MAP under the prior $p_{X}(x)=\exp (-\phi(x)) / C_{\phi}$ ", where

$$
C_{\phi}:=\int_{\mathbb{R}^{n}} \exp (-\phi(x)) d x
$$

In particular, when $\phi(x)=\|x\|_{1}$, a possible interpretation of (I.3) is MAP denoising under a Laplacian prior on $x$ and white Gaussian noise.

The main objective of this paper is to highlight the fact that while one Bayesian interpretation of the penalized least-squares estimator (I.3) with penalty function $\phi(x)$ is the MAP estimator $\psi_{\text {MAP }}(y)$ with prior $p_{X}(x)=$ $\exp (-\phi(x)) / C_{\phi}$, there can be other admissible Bayesian interpretations.

We focus on white Gaussian denoising and show that for any prior $P_{X}$ and any noisy observation $y \in \mathbb{R}^{n}$, the MMSE estimate $\psi_{\text {MMSE }}(y)$ under the prior $P_{X}$ is the solution of a penalized least-squares problem (I.3) with an appropriate penalty function $\phi_{\text {MMSE }}(x)$. Thus, the problem (I.3) with penalty $\phi_{\text {MMSE }}(x)$ can equally be interpreted as: a) the MAP estimator $\tilde{\psi}_{M A P}(y)$ with a prior associated to the pdf $\tilde{p}_{X}(x)=\exp \left(-\phi_{\mathrm{MMSE}}(x)\right) / C_{\phi_{\mathrm{MMSE}}}$; or b) the MMSE estimator with prior $P_{X}$. In general $d P_{X}(x) \neq \tilde{p}_{X}(x) d x$.

\footnotetext{
${ }^{1}$ This interpretation only makes sense if $C_{\phi}<\infty$ is integrable. Otherwise some authors refer to a "non-informative prior".
} 


\section{MAIN RESUlTS}

From now on we focus on Gaussian denoising: $B \in \mathbb{R}^{n}$ is a centered normal Gaussian variable with law $P_{B}=\mathcal{N}\left(0, \mathbf{I}_{n}\right)$ and pdf $p_{B}(b) \propto \exp \left(-\|b\|_{2}^{2} / 2\right)$. We let $X \in \mathbb{R}^{n}$ be a random variable independent of $B$, with law $P_{X}$. The probability distribution of the noisy observation $Y=X+B$ has a pdf

$$
p_{Y}(y):=p_{B} \star P_{X}(y)=\int_{\mathbb{R}^{n}} p_{B}(y-x) d P_{X}(x)
$$

which is sometimes refered to as the evidence of the observation $y$. When $P_{X}$ is associated to a pdf $p_{X}(x)$, the evidence is given by a standard convolution between pdfs $p_{Y}=p_{B} \star p_{X}$. Even when $P_{X}$ is not associated to a pdf, $p_{Y}$ infinitely differentiable, i.e., $p_{Y} \in C^{\infty}\left(\mathbb{R}^{n}\right)$.

In this setting, using techniques going back to Stein's unbiased risk estimator [9], [1], one can express the MMSE estimator as [8]

$$
\psi_{\mathrm{MMSE}}(y)=y+\frac{1}{p_{Y}(y)}\left[\frac{\partial}{\partial y_{i}} p_{Y}(y)\right]_{i=1}^{n}=y+\nabla \log p_{Y}(y) .
$$

All vectors $u \in \mathbb{R}^{n}$, such as the gradient $\nabla \log p_{Y}(y) \in \mathbb{R}^{n}$, are in column form. Their transpose $u^{T}$ is in row form.

Next we study whether $\psi_{\text {MMSE }}$ can also be written as the optimum of an optimization problem of the MAP type (I.3), with an appropriate choice of $\phi$. Namely, we investigate when $\psi_{\text {MMSE }}$ can be identified with the proximity operator [2] of a function $\phi$, where we recall the definition

$$
\operatorname{prox}_{\phi}(y):=\arg \min _{z \in \mathbb{R}^{n}}\left\{\frac{1}{2}\|y-z\|_{2}^{2}+\phi(z)\right\} .
$$

Rereading Equation (I.2) the MAP estimator (with prior $p_{X}(x)$ ) can be written as $\psi_{\text {MAP }}=\operatorname{prox}_{\phi_{\text {MAP }}}$ where

$$
\phi_{\text {MAP }}(x):=-\log p_{X}(x) .
$$

For smooth $\phi$ we have the implicit characterization [2]

$$
\operatorname{prox}_{\phi}(y):=y-\nabla \phi\left[\operatorname{prox}_{\phi}(y)\right], \quad \forall y \in \mathbb{R}^{n} .
$$

Comparing (II.2) with (II.5), we see that if $\psi_{\mathrm{MMSE}}=\operatorname{prox}_{\phi}$ then

$$
\nabla \phi\left[\psi_{\text {MMSE }}(y)\right]=-\nabla \log p_{Y}(y), \quad \forall y \in \mathbb{R}^{n} .
$$

Indeed, the relation (II.6) characterizes all functions $\phi$ such that $\psi_{\mathrm{MMSE}}=\operatorname{prox}_{\phi}$, thanks to the following lemma.

Lemma II.1. Let $X P_{X}, B \sim P_{B} \mathcal{N}(0, \mathbf{I})$ be independent random variables in $\mathbb{R}^{n}$. Assume that there is no pair $v \in \mathbb{R}^{n}, c \in \mathbb{R}$ such that $\langle X, v\rangle=c$ with probability one. Then the MMSE estimator $y \mapsto \psi_{M M S E}(y)$ has the following properties:

1) it is one-to-one from $\mathbb{R}^{n}$ onto Im $\psi_{M M S E} \subset \mathbb{R}^{n}:$ for any pair $y, y^{\prime} \in \mathbb{R}^{n}$, if $\psi_{M M S E}(y)=\psi_{M M S E}\left(y^{\prime}\right)$ then $y=y^{\prime}$.

2) it is $C^{\infty}\left(\mathbb{R}^{n}\right)$; so is its inverse $\psi_{M M S E}^{-1}: \operatorname{Im} \psi_{M M S E} \rightarrow \mathbb{R}^{n}$.

3) when $n=1$ we further have that $\psi_{M M S E}$ is increasing.

The proof is in Appendix A. Note that the probability distribution $P_{X}$ in Lemma II.1 can be almost arbitrary, provided that there is no lower-dimensional affine space of $\mathbb{R}^{n}$ to which $X$ belongs almost surely. In particular, $P_{X}$ need not be separable. In light of this lemma, (II.6) is equivalent to

$$
\nabla \phi(z)=-\nabla \log p_{Y}\left[\psi_{\mathrm{MMSE}}^{-1}(z)\right], \quad \forall z \in \operatorname{Im} \psi_{\mathrm{MMSE}} .
$$

As shown by our main theorem (the proof is in Appendix B), this equation is satisfied by the function $\phi_{\mathrm{MMSE}}$ : $\mathbb{R}^{n} \rightarrow \mathbb{R} \cup\{+\infty\}$ defined as:

$$
\phi_{\mathrm{MMSE}}(x):= \begin{cases}-\frac{1}{2}\left\|\psi_{\mathrm{MMSE}}^{-1}(x)-x\right\|_{2}^{2} & -\log p_{Y}\left[\psi_{\mathrm{MMSE}}^{-1}(x)\right] \\ & \text { for } x \in \operatorname{Im} \psi_{\mathrm{MMSE}} \\ +\infty, & \text { for } x \notin \operatorname{Im} \psi_{\mathrm{MMSE}}\end{cases}
$$


Theorem II.2. Let $X P_{X}, B \sim P_{B}=\mathcal{N}(0, \mathbf{I})$ be independent random variables in $\mathbb{R}^{n}$. Assume that there is no lower-dimensional affine space of $\mathbb{R}^{n}$ to which $X$ belongs almost surely. Then prox ${ }_{\phi_{\text {MMSE }}}=\psi_{\text {MMSE }}$ and:

1) the function $\phi_{M M S E}$ is $C^{\infty}$ on its domain Im $\psi_{M M S E}$;

2) for every $y \in \mathbb{R}^{n}$, the vector $\psi_{M M S E}(y)=\operatorname{prox}_{\phi_{\text {MMSE }}}(y)$ is the unique global minimum, as well as the unique stationary point of the function $x \mapsto \frac{1}{2}\|y-x\|^{2}+\phi_{M M S E}(x)$;

3) for every $y \in \mathbb{R}^{n}$, we have $\phi_{M M S E}(y) \geq-\log p_{Y}(y)$;

4) we have $C_{\phi_{\text {MSSE }}}=\int_{\mathbb{R}^{n}} \exp \left(-\phi_{\text {MMSE }}(x)\right) d x<\infty$.

Therefore, the MMSE estimator with prior $P_{X}$ and white Gaussian noise is also the MAP estimator with the prior which pdf is $\tilde{p}_{X}(x)=\exp \left(-\phi_{M M S E}(x)\right) / C_{\phi_{\text {MMSE }}}$.

Remark II.1. Note that $\psi(y)$ is not only the unique global minimum of $x \mapsto \frac{1}{2}\|y-x\|^{2}+\phi_{\text {MMSE }}(x)$ : it is also its unique stationary point. This is much stronger: this means that descent algorithms used to solve the optimization problem (I.3) with $\phi=\phi_{\text {MMSE }}$ cannot be trapped in a spurious local minimum.

Remark II.2. When $X$ belongs with probability one to a lower-dimensional affine space $V \subset \mathbb{R}^{n}$, we have $\operatorname{Im} \psi_{\text {MMSE }} \subset V$. Letting $V$ be the smallest such affine space, the restriction of $\psi_{\text {MMSE }}$ to $V$ still has a well defined $C^{\infty}$ inverse $\psi_{\text {MMSE }}^{-1}: \operatorname{Im} \psi_{\text {MMSE }} \rightarrow V$ which can be used to define $\phi_{\text {MMSE }}$ as in (II.7) and to generalize Theorem II.2 to an arbitrary prior $P_{X}$.

\section{WORKED EXAMPLE}

Let us illustrate Theorem II.2 with a simple example: we consider the one-dimensional $(n=1)$ mixture of two Gaussians prior on the unknown noiseless data $x$,

$$
p_{X}(x):=p \cdot \frac{e^{-\frac{x^{2}}{2 \sigma_{0}^{2}}}}{\sqrt{2 \pi \sigma_{0}^{2}}}+(1-p) \cdot \frac{e^{-\frac{x^{2}}{2 \sigma_{1}^{2}}}}{\sqrt{2 \pi \sigma_{1}^{2}}},
$$

where $p \in(0,1)$ and $0<\sigma_{0}<\sigma_{1}$. The evidence of the observed noisy data $Y=X+B$ with $B \sim \mathcal{N}(0,1)$ is then

$$
p_{Y}(y)=p \cdot \frac{e^{-\frac{y^{2}}{2\left(\sigma_{0}^{2}+1\right)}}}{\sqrt{2 \pi\left(\sigma_{0}^{2}+1\right)}}+(1-p) \cdot \frac{e^{-\frac{y^{2}}{2\left(\sigma_{1}^{2}+1\right)}}}{\sqrt{2 \pi\left(\sigma_{1}^{2}+1\right)}},
$$

hence

$$
p_{Y}^{\prime}(y)=-y \cdot\left\{p \cdot \frac{e^{-\frac{y^{2}}{2\left(\sigma_{0}^{2}+1\right)}}}{\sqrt{2 \pi\left(\sigma_{0}^{2}+1\right)^{3}}}+(1-p) \cdot \frac{e^{-\frac{y^{2}}{2\left(\sigma_{1}^{2}+1\right)}}}{\sqrt{2 \pi\left(\sigma_{1}^{2}+1\right)^{3}}} \cdot\right\}
$$

By straightforward computations, we obtain

$$
\begin{aligned}
\psi_{\text {MMSE }}(y) & =y \cdot \frac{\frac{\sigma_{0}^{2}}{\sigma_{0}^{2}+1}+\frac{\sigma_{1}^{2}}{\sigma_{1}^{2}+1} \cdot a e^{b y^{2}}}{1+a e^{b y^{2}}} \text { with } \\
a & :=\frac{1-p}{p} \sqrt{\frac{\sigma_{0}^{2}+1}{\sigma_{1}^{2}+1}}, \quad b=\frac{1}{\sigma_{0}^{2}+1}-\frac{1}{\sigma_{1}^{2}+1} \in(0,1) .
\end{aligned}
$$

The limiting case $\sigma_{0}^{2} \rightarrow 0$ corresponds to the so called Bernoulli-Gaussian prior (see, e.g., [11]): the value $x=0$ is drawn with probability $p>0$, hence vectors with i.i.d. entries distributed according to $p_{X}$ are typically sparse. The MMSE estimator takes a simplified form [10] when $\sigma_{0}^{2} \rightarrow 0$

$$
\psi_{\mathrm{MMSE}}(y)=y \cdot \frac{\sigma_{1}^{2}}{\sigma_{1}^{2}+1} \cdot \frac{a e^{b y^{2}}}{1+a e^{b y^{2}}} .
$$

We illustrate in Figure 1 the case $p=0.9, \sigma_{0}^{2} \rightarrow 0, \sigma_{1}^{2}=10$. Figure 1(a) shows $\psi_{\text {MMSE }}$ (solid line) and its inverse $\psi_{\text {MMSE }}^{-1}$ (dashed line). The latter does not seem to have an analytic expression. Figure 1(b) shows $\phi_{\mathrm{MAP}}(x)=-\log p_{X}(x)$ (dotted line), $-\log p_{Y}(x)$ (dashed line) and the penalty function $\phi_{\mathrm{MMSE}}(x)$ (solid line). While the penalty function $\phi_{\mathrm{MMSE}}(x)$ does not seem to admit an analytic expression, one can obtain an analytic expression for $\phi_{\mathrm{MMSE}}\left[\psi_{\mathrm{MMSE}}(y)\right]=-\frac{1}{2}\left\|y-\psi_{\mathrm{MMSE}}(y)\right\|_{2}^{2}-\log p_{Y}(y)$. The explicit analytic expression which is long and rather uninteresting- was used to plot $\phi_{\text {MMSE }}(x)$ on Figure 1(a) using the parameterized curve 
(a) MMSE estimator and its inverse $\left(\mathrm{p}=0.99 \sigma_{1}^{2}=10\right)$

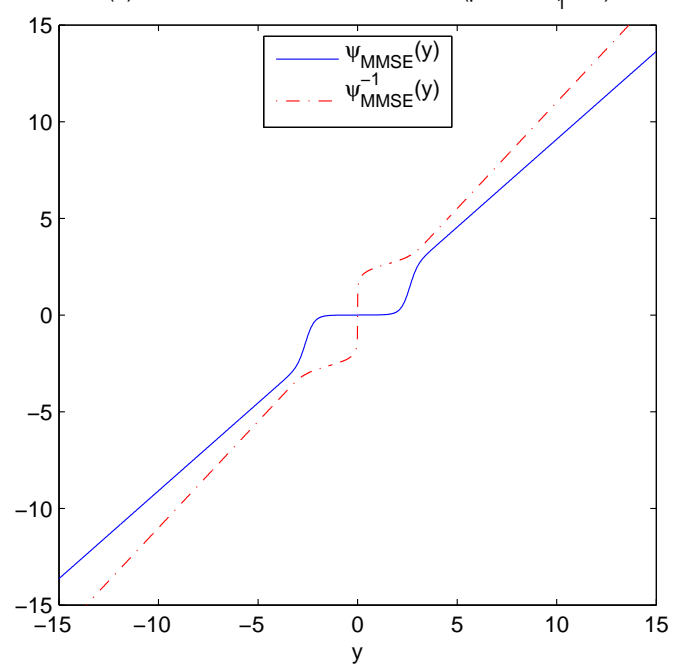

(b) Penalty functions $\left(\mathrm{p}=0.99 \sigma_{1}^{2}=10\right)$

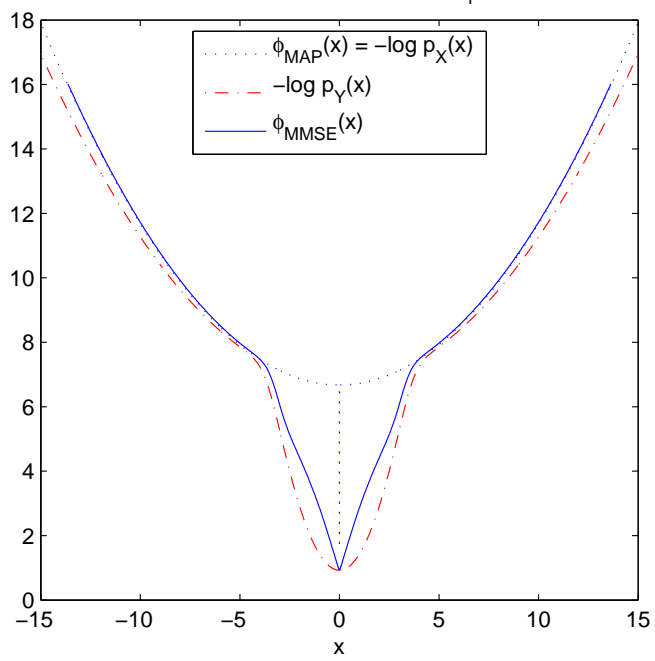

Fig. 1. Left: MMSE estimator $\psi_{\text {MMSE }}(y)$ (solid line) and its inverse $\psi_{\text {MMSE }}^{-1}(y)$ (dashed line), in the Bernoulli-Gaussian case, $p=0.9$, $\sigma_{0}^{2} \rightarrow 0, \sigma_{1}^{2}=10$. Right: MAP penalty $\phi_{\mathrm{MAP}}(x)=-\log p_{X}(x)$ (dotted line), negative log-evidence $\left(-\log p_{Y}(x)\right)($ dashed line) and MMSE penalty $\phi_{\text {MMSE }}(x)$ (solid line).

$y \mapsto\left(\psi_{\text {MMSE }}(y), \phi_{\text {MMSE }}\left[\psi_{\text {MMSE }}(y)\right]\right)$. Observing on Figure $1(b)$ the plot of $\phi_{\text {MMSE }}(x)$ for the above Bernoulli-Gaussian prior yields a number of observations.

1) For small $x$, the penalty $\phi_{\mathrm{MMSE}}(x)$ is approximately shaped as the absolute value: $\phi_{\mathrm{MMSE}}(x) \approx c|x|$ for some constant $c$. This is tempered by the fact that $\phi_{\mathrm{MMSE}}(x)$ is $C^{\infty}$, thus, unlike $|x|$, it must be smooth at zero.

2) The penalty $\phi_{\mathrm{MMSE}}(x)$ is unimodal (it is decreasing until its global minimum, then increasing) but it is not convex.

The second observation could seem surprising given that Theorem II.2 guarantees the uniqueness of the global minimizer / stationary point of $x \mapsto \frac{1}{2}\|y-x\|^{2}+\phi_{\mathrm{MMSE}}(x)$. However, this property is not a characteristic of convex penalties. As a matter of fact, a function $f: \mathbb{R} \rightarrow \mathbb{R}$ (i.e., in the case $n=1$ ) can be written $f=\operatorname{prox}_{g}$ with $g$ a proper lower semi-continuous convex function from $\mathbb{R}$ to $\mathbb{R} \cup\{+\infty\}$ if, and only if, the function $f$ is increasing and non-expansive [2, Proposition 2.4]:

Definition III.1. A function $f: \mathbb{R} \rightarrow \mathbb{R}$ is non-expansive if $\left|f\left(y^{\prime}\right)-f(y)\right| \leq\left|y^{\prime}-y\right|$ for all $y, y^{\prime}$. When $f$ is differentiable, it is non-expansive if and only if $\left|f^{\prime}(y)\right| \leq 1$ for all $y$.

By Lemma II.1, in dimension $n=1$, the MMSE estimator $\psi_{\text {MMSE }}$ is increasing for any prior $P_{X}$. However, for certain priors $P_{X}$, it can indeed be proved to be expansive (see the proof in Appendix C):

Proposition III.2. Assume that $X$ has a symmetric $p d f\left[\forall x \in \mathbb{R}, p_{X}(-x)=p_{X}(x)\right]$ and that there exists $\varepsilon>0$ such that $p_{X}(x)=0$ for all $x$ with $|x|<1+\varepsilon$. Then the penalty $\phi_{M M S E}$ cannot be convex.

\section{DiscusSiON}

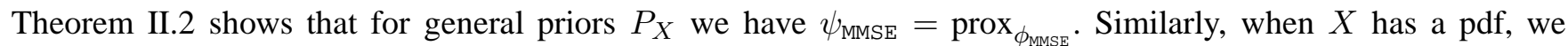
have $\psi_{\text {MAP }}=\operatorname{prox}_{\phi_{\text {MAP }}}$, where for a given prior the MAP penalty $\phi_{\text {MAP }}(x)$ has the simple expression (II.4) while the MMSE penalty $\phi_{\text {MMSE }}(x)$ has the much more intricate definition (II.7).

For Gaussian priors $P_{X}=\mathcal{N}(0, \Sigma)$, the MMSE estimator is the Wiener filter, which is also the MAP and the minimum mean square linear estimator [5], so $\phi_{\mathrm{MMSE}}=\phi_{\mathrm{MAP}}$ (up to a constant additive term).

However, for most priors with a pdf $p_{X}(x)$, the MMSE estimator does not coincide with the MAP estimator (i.e., $\psi_{\mathrm{MMSE}} \neq \psi_{\mathrm{MAP}}$ ), hence $\phi_{\mathrm{MMSE}} \neq \phi_{\mathrm{MAP}}$ (even up to a constant additive term). Indeed, by Theorem II.2, the penalty $\phi_{\text {MMSE }}(x)$ defined in (II.7) has a number of specific properties. Therefore, if $\phi_{\text {MAP }}(x)=-\log p_{X}(x)$ fails to satisfy one of these properties, then the identity $\phi_{\mathrm{MMSE}}(x)=\phi_{\mathrm{MAP}}(x)+c$ (for some constant $c \in \mathbb{R}$ and all $x \in \mathbb{R}^{n}$ ) cannot be satisfied. 
For example, generalized Gaussian priors $p_{X}(x) \propto \exp \left(-\alpha\|x\|_{p}^{p}\right)$ with $0<p \leq 1$ are not smooth at $x=0$, hence they are not $C^{\infty}$ : as a result for such priors there is not even any pair $a, b \in \mathbb{R}$ such that $\phi_{\text {MMSE }}(x)=a+b \cdot \phi_{\text {MAP }}(x)$ for all $x$.

One may also wonder whether a reciprocal to Theorem II.2 is possible: given a penalty function $\phi(x)$, does there exist a prior $P_{X}$ such that the MMSE estimator $\psi_{\text {MMSE }}$ with this prior is associated to the penalty $\phi_{\text {MMSE }}(x)=\phi(x)$ (up to a constant additive term) ? When this prior exists, can we characterize it in terms of the penalty function $\phi$ ? Even though one can always define the tentatively associated "MMSE estimator" $\psi(y)=\operatorname{prox}_{\phi}(y)$, the main difficulty is to understand when there exists a probability measure $P_{X}$ such that $\psi(y)-y=\nabla \log \left(p_{B} \star P_{X}\right)(y)$. This combined integration and Gaussian deconvolution problem often does not admit a solution, for example: when $\psi$ is not one to one; when $\phi(x)$ is not sufficiently smooth.

\section{CONCLUSION AND PERSPECTIVES}

We proved that the MMSE estimator for Gaussian denoising with any prior can be written as the MAP estimator with a possibly different prior (and that the MAP estimator with certain priors can be interpreted as a MMSE estimator with a possibly different prior). These results, in conjunction with Nikolova's highlighting of model distortions brought by MAP estimation [7], indicate that one should be cautious when interpreting penalized least squares regression schemes in terms of priors:

- If the unknown noiseless data $x$ follows a prior with pdf $p_{X}(x) \propto \exp (-\phi(x))$ and if we choose the MAP as a criterion for estimating it, then the resulting denoising scheme leads to penalized least squares regression with penalty $\phi(x)$. This MAP estimator may however have poor denoising performance ${ }^{2}$ for this type of data [7].

- In practice, the choice of penalized least squares regression with penalty $\phi(x)$ is seldomly associated to the belief that the unknown noiseless data follows a prior with pdf $p_{X}(x) \propto \exp (-\phi(x))$. Instead, it rather stems from the need for numerical efficiency and the empirical observation that it achieves good denoising performance for the considered class of data.

By definition, optimum denoising (as measured by the mean squares error) is achieved by the MMSE estimator. As shown in this paper, the latter is indeed always associated to a penalized least squares scheme ${ }^{3}$. This sheds a new light on the popularity of such schemes for Gaussian denoising.

Quite obviously, the denoising performance of penalized least squares regression with a given penalty $\phi(x)$ heavily depends on the prior $P_{X}$ underlying the unknown noiseless data. We focused in this paper on the case where the penalized least squares regression estimator $\psi(y)=\operatorname{prox}_{\phi}(y)$ coincides with the MMSE estimator: its denoising performance $\mathbb{E}\left(\left\|\operatorname{prox}_{\phi}(Y)-X\right\|_{2}^{2}\right)$ is optimum. An interesting open problem related to the results of this paper would be to understand for which priors $P_{X}$ we obtain "good" denoising performance with $\psi(y)=\operatorname{prox}_{\phi}(y)$, i.e., when the denoising performance is bounded by a constant $C>1$ times the optimum performance.

One can imagine concrete applications of the results presented here for certain priors: in general the MMSE estimator $\psi_{\text {MMSE }}(y)$ is a priori expressed as an intractable high-dimensional integral; however, if the penalty function $\phi_{\text {MMSE }}(x)$ admits a simple expression amenable to efficient numerical optimization (e.g., convex optimization), then the MMSE estimator can be computed efficiently. Developping such approaches requires a more in-depth understanding of the properties of penalty functions $\phi_{\mathrm{MMSE}}(x)$ obtained through Theorem II.2. Of particular interest would be the construction of explicit examples where $\phi_{\text {MMSE }}(x)$ is "simple" while $p_{Y}(y)$ involves an intractable integral.

Another interesting perspective is to obtain alternate statistical interpretations of a larger class of penalized least squares regression estimators (e.g., with non-smooth $\phi(x)$ such as those leading to sparse estimates). As remarked above, the lack of smoothness makes it impossible to interpret such estimators in terms of a MMSE estimator, however one may seek interpretations that leave the strict Bayesian framework: for example, one may wish to obtain an interpretation as the optimum of a hybrid Bayesian cost function $\min _{\psi}\{\mathbb{E} \mathcal{C}(\psi(X+B), X)+\mathbf{K}(\psi)\}$ where the term $\mathbf{K}(\cdot)$ forces the function $\psi$ to be in some function class. Eventually, one may also wish to extend theses results to ill-posed linear inverse problems of the type $y=\mathbf{A} x+b$, and to deal with non-Gaussian noise.

\footnotetext{
${ }^{2}$ Even though, as shown in this paper, this MAP scheme can sometimes be interpreted as an MMSE estimator with a different prior, this re-interpretation does not alter the denoising scheme nor its denoising performance.

${ }^{3}$ Yet, the associated penalized least squares problem may not be more computationally tractable than the original MMSE.
} 


\section{ACKNOWLEDGEMENTS}

This existence of this paper owes much to several discussions with Mike Davies about the Bayesian "interpretation" of sparse regularization, as well as intense discussions with Jérôme Idier on the same topic during the second French Spring School of Inverse Problems in Signal Processing, held in the beautiful mediterranean island of Porquerolles in the spring of 2010. The author is very thankful to Mike, Jérôme, and to the organizers of the Spring school for these passionate discussions, and would also like to thank Jean-Christophe Pesquet, who provided his insight on proximity operators, as well as Patrick Perez and Miki Elad, whose comments on a draft version of this paper were precious. Last, the comments of the three anonymous reviewers were very helpful to improve the final version of the paper.

\section{APPENDIX}

\section{A. Proof of Lemma II.1}

Lemma A.1. Denote $\psi_{M M S E}(y)=\left(\psi_{M M S E}^{i}(y)\right)_{i=1}^{n}$ where $\psi_{M M S E}^{i}: \mathbb{R}^{n} \rightarrow \mathbb{R}$ is scalar valued. Under the assumptions of Lemma II.1, the $n \times n$ Jacobian matrix $J\left[\psi_{M M S E}\right](y):=\left(\frac{\partial}{\partial y_{j}} \psi_{M M S E}^{i}(y)\right)_{i j}$ satisfies the identity

$$
J\left[\psi_{M M S E}\right](y)=\left(\delta_{i j}+\frac{\partial^{2} \log p_{Y}(y)}{\partial y_{i} \partial y_{j}}\right)_{i j}=\mathbf{I}+\nabla^{2} \log p_{Y}(y)
$$

and is symmetric positive definite:

$$
\left\langle v, J\left[\psi_{M M S E}\right](y) \cdot v\right\rangle>0, \quad \forall y \in \mathbb{R}^{n}, v \neq 0 .
$$

Proof: Without loss of generality we consider a unit norm vector $\|v\|_{2}=1$. For brevity we omit the dependency in the variable $y$ when possible. First, by (I.2) we have

$$
\psi_{\text {MMSE }}(y)=y+\nabla \log p_{Y}(y)=y+\nabla p_{Y}(y) / p_{Y}(y)
$$

hence

$$
J\left[\psi_{\mathrm{MMSE}}\right]=\mathbf{I}+\nabla^{2} \log p_{Y}=\mathbf{I}+\frac{\nabla^{2} p_{Y}}{p_{Y}}-\frac{\nabla p_{Y} \cdot\left(\nabla p_{Y}\right)^{T}}{\left[p_{Y}\right]^{2}}
$$

and

$$
\left\langle J\left[\psi_{\mathrm{MMSE}}\right] \cdot v, v\right\rangle=\frac{p_{Y}^{2}+p_{Y}\left\langle\nabla^{2} p_{Y} \cdot v, v\right\rangle-\left\langle\nabla p_{Y}, v\right\rangle^{2}}{p_{Y}^{2}} .
$$

We will now prove that the numerator in (A.3) is positive for all $y$. Since $p_{B}(b) \propto e^{-\|b\|_{2}^{2} / 2}$, we have

$$
\begin{aligned}
\nabla p_{B}(b) & =(-b) \cdot p_{B}(b), \\
\nabla^{2} p_{B}(b) & =\left(b b^{T}-\mathbf{I}\right) \cdot p_{B}(b) .
\end{aligned}
$$

Since $p_{Y}=p_{B} \star P_{X}, \nabla p_{Y}=\nabla p_{B} \star P_{X}, \nabla^{2} p_{Y}=\nabla^{2} p_{B} \star P_{X}$ this yields

$$
\begin{aligned}
p_{Y} & =\int p_{B}(y-x) d P_{X}(x) \\
\left\langle\nabla p_{Y}, v\right\rangle & =\int(-\langle y-x, v\rangle) \cdot p_{B}(y-x) d P_{X}(x) \\
\left\langle\nabla^{2} p_{Y} \cdot v, v\right\rangle & =\int\left(\langle y-x, v\rangle^{2}-1\right) \cdot p_{B}(y-x) d P_{X}(x)
\end{aligned}
$$

hence

$$
\begin{aligned}
p_{Y}\left\langle\nabla^{2} p_{Y} \cdot v, v\right\rangle=\iint & \left(\langle y-x, v\rangle^{2}-1\right) \\
\cdot & p_{B}(y-x) p_{B}\left(y-x^{\prime}\right) d P_{X}(x) d P_{X}\left(x^{\prime}\right)
\end{aligned}
$$


The above expression is also valid is we exchange the role of the integration variables $b$ and $b^{\prime}$, hence by taking the average of these two equal expressions we obtain

$$
\begin{aligned}
p_{Y}\left\langle\nabla^{2} p_{Y} \cdot v, v\right\rangle=\iint & {\left[\frac{\langle y-x, v\rangle^{2}+\left\langle y-x^{\prime}, v\right\rangle^{2}}{2}-1\right] } \\
& \cdot p_{B}(y-x) p_{B}\left(y-x^{\prime}\right) d P_{X}(x) d P_{X}\left(x^{\prime}\right)
\end{aligned}
$$

Similarly we can write

$$
\begin{aligned}
p_{Y}^{2}= & \iint p_{B}(y-x) p_{B}\left(y-x^{\prime}\right) d P_{X}(x) d P_{X}\left(x^{\prime}\right) \\
\left\langle\nabla p_{Y}, v\right\rangle^{2}=\iint\langle y-x, v\rangle\left\langle y-x^{\prime}, v\right\rangle & \int p_{B}(y-x) p_{B}\left(y-x^{\prime}\right) d P_{X}(x) d P_{X}\left(x^{\prime}\right)
\end{aligned}
$$

Overall, the numerator of the right hand side in (A.3) becomes

$$
\iint \frac{\left\langle x^{\prime}-x, v\right\rangle^{2}}{2} p_{B}(y-x) p_{B}\left(y-x^{\prime}\right) d P_{X}(x) d P_{X}\left(x^{\prime}\right)
$$

Now, since there is no $c$ such that $\langle X, v\rangle=c$ with probability one, there exists $x_{1}, x_{2} \in \mathbb{R}^{n}, d=\left\langle x_{2}-x_{1}, v\right\rangle \neq 0$, such that the Euclidean balls $B_{i}=B\left(x_{i}, d / 3\right) \subset \mathbb{R}^{n}$, have positive probability $P_{X}\left(B_{i}\right)>0$. For $\left(x, x^{\prime}\right) \in B_{1} \times B_{2}$ the function $g\left(x, x^{\prime}\right):=\frac{\left\langle x^{\prime}-x, v\right\rangle^{2}}{2} p_{B}(y-x) p_{B}\left(y-x^{\prime}\right)$ is bounded from below by some constant $\eta>0$, hence the integral in (A.4) is bounded from below by

$$
\iint_{B_{1} \times B_{2}} g\left(x, x^{\prime}\right) d P_{X}(x) d P_{X}\left(x^{\prime}\right) \geq \eta \cdot P_{X}\left(B_{1}\right) P_{X}\left(B_{2}\right)>0 .
$$

We conclude that $\left\langle J\left[\psi_{\text {MMSE }}\right] \cdot v, v\right\rangle>0$.

We are now equipped to prove Lemma II.1.

Proof of Lemma II.1: We let the reader check that $p_{Y}$ cannot vanish. Since it is $C^{\infty}, \psi_{\mathrm{MMSE}}$ is also $C^{\infty}$. To prove that $\psi_{\text {MMSE }}$ is one-to-one, we proceed by contradiction, assuming that $\psi_{\text {MMSE }}(y)=\psi_{\text {MMSE }}\left(y^{\prime}\right)$ while $y^{\prime} \neq y$. We define $v:=\left(y^{\prime}-y\right) /\left\|y^{\prime}-y\right\|_{2}$ and the function $f: t \mapsto f(t):=\left\langle v, \psi_{\text {MMSE }}(y+t v)\right\rangle \in \mathbb{R}$. Since the function $f$ is smooth and $f(0)=f\left(\left\|y^{\prime}-y\right\|_{2}\right)$, by Rolle's theorem the derivative of $f$ must vanish for some $0<t<\left\|y^{\prime}-y\right\|_{2}$. However by Lemma A.1 we have $f^{\prime}(t)=\left\langle v, J\left[\psi_{\mathrm{MMSE}}\right](y+t v) \cdot v\right\rangle>0$ which yields a contradiction. Therefore, the inverse function $\psi_{\text {MMSE }}^{-1}$ exists as claimed. The fact that it is also $C^{\infty}$ follows from the positivity of the Jacobian of $\psi_{\text {MMSE }}$ and the inverse function theorem.

\section{B. Proof of Theorem II.2}

The fact that $\phi_{\text {MMSE }}$ is $C^{\infty}$ on $\operatorname{Im} \psi_{\text {MMSE }}$ is a straightforward consequence of its definition (II.7) and of the fact that $p_{Y}$ as well as $\psi_{\text {MMSE }}^{-1}$ are $C^{\infty}$ (Lemma II.1). We wish to check that the proximity operator of $\phi_{\text {MMSE }}$ defined by (II.7) is indeed $\psi_{\mathrm{MMSE}}$. The definition of $\phi_{\text {MMSE }}(x)$ for $x \notin \operatorname{Im} \psi_{\text {MMSE }}$ ensures that prox $\phi_{\text {MMSE }}$ takes its values in $\operatorname{Im} \psi_{\text {MMSE }}$. We let the reader check that a consequence of Lemma A.1 is that the $\operatorname{set} \operatorname{Im} \psi_{\text {MMSE }}$ is open. For brevity we denote $q(y)=\log p_{Y}(y)$ and

$$
\begin{aligned}
g(u) & :=\frac{1}{2}\left\|y-\psi_{\mathrm{MMSE}}(u)\right\|_{2}^{2}+\phi_{\mathrm{MMSE}}\left[\psi_{\mathrm{MMSE}}(u)\right] \\
& =\frac{1}{2}\left\|\psi_{\mathrm{MMSE}}(u)-y\right\|_{2}^{2}-\frac{1}{2}\|\nabla q(u)\|_{2}^{2}-q(u) .
\end{aligned}
$$

Since $J\left[\psi_{\mathrm{MMSE}}\right](u)=\mathbf{I}+\nabla^{2} q(u)$ (Lemma A.1) and $\psi_{\mathrm{MMSE}}(u)=u+\nabla q(u)$ (Equation (II.2)), we obtain

$$
\begin{aligned}
\nabla g(u)= & J\left[\psi_{\text {MMSE }}\right](u) \cdot\left[\psi_{\text {MMSE }}(u)-y\right] \\
& -\nabla^{2} q(u) \cdot \nabla q(u)-\nabla q(u) \\
= & J\left[\psi_{\text {MMSE }}\right](u) \cdot\left[\psi_{\text {MMSE }}(u)-y-\nabla q(u)\right]
\end{aligned}
$$




$$
=J\left[\psi_{\mathrm{MMSE}}\right](u) \cdot[u-y]
$$

Now consider $f_{v}(t):=g(y+t v)$ with $v \neq 0$ an arbitrary vector. Its derivative is

$$
\begin{aligned}
f_{v}^{\prime}(t) & =\langle\nabla g(y+t v), v\rangle=\left\langle J\left[\psi_{\mathrm{MMSE}}\right](y+t v) \cdot t v, v\right\rangle \\
& =t \cdot\left\langle J\left[\psi_{\mathrm{MMSE}}\right](y+t v) \cdot v, v\right\rangle
\end{aligned}
$$

which, by Lemma A.1, has the sign of $t$, showing that $f_{v}$ admits its strict global minimum at $t=0$. Since this is true for any choice of $v$ it follows that $g$ has no stationary point other that $u=y$, and that $g(u)>g(y)$ whenever $u \neq v$, that is to say $x \mapsto \frac{1}{2}\|y-x\|_{2}^{2}+\phi_{\mathrm{MMSE}}(x)$ admits a unique global minimum at $x=\psi_{\mathrm{MMSE}}(y)$. To conclude, since $\psi_{\mathrm{MMSE}}(y)=\operatorname{prox}_{\phi_{\mathrm{MMSE}}}(y)$, we have for any $y$

$$
\begin{aligned}
\phi_{\text {MMSE }}(y) & =\frac{1}{2}\|y-y\|_{2}^{2}+\phi_{\text {MMSE }}(y) \\
& \geq \frac{1}{2}\left\|y-\psi_{\text {MMSE }}(y)\right\|_{2}^{2}+\phi_{\text {MMSE }}\left[\psi_{\text {MMSE }}(y)\right] \\
& =-\log p_{Y}(y) .
\end{aligned}
$$

As a result $0 \leq \exp \left(-\phi_{\text {MMSE }}(y)\right) \leq p_{Y}(y)$, and since $p_{Y}(y)$ is integrable so is $\exp \left(-\phi_{\text {MMSE }}(y)\right)$.

\section{Proof of Lemma III.2}

Thanks to (A.4), and since both $p_{B}$ and $p_{X}$ are symmetric, the numerator of (A.3) for $y=0$ reads

$$
\begin{aligned}
\iint & \frac{\left(x^{\prime}-x\right)^{2}}{2} \cdot p_{B}(-x) p_{B}\left(-x^{\prime}\right) p_{X}(x) p_{X}\left(x^{\prime}\right) d x d x^{\prime} \\
= & \int \frac{x^{2}}{2} \cdot p_{B}(x) p_{X}(x) d x \cdot \int p_{B}\left(x^{\prime}\right) p_{X}\left(x^{\prime}\right) d x^{\prime} \\
& +\int \frac{\left(x^{\prime}\right)^{2}}{2} \cdot p_{B}\left(x^{\prime}\right) p_{X}\left(x^{\prime}\right) d x^{\prime} \cdot \int p_{B}(x) p_{X}(x) d x \\
& \quad-\int x^{\prime} \cdot p_{B}\left(x^{\prime}\right) p_{X}\left(x^{\prime}\right) d x^{\prime} \cdot \int x \cdot p_{B}(x) p_{X}(x) d x \\
= & \int x^{2} \cdot p_{B}(x) p_{X}(x) d x \cdot \int p_{B}\left(x^{\prime}\right) p_{X}\left(x^{\prime}\right) d x^{\prime}
\end{aligned}
$$

Since $p_{Y}(y)=\int p_{B}(x) p_{X}(x) d x$, inserting the above expression in (A.3) for $y=0$ and using that $p_{X}(x)=0$ for $|x|<1+\varepsilon$ we obtain

$$
\begin{aligned}
& \psi_{\text {MMSE }}^{\prime}(0)=\frac{\int x^{2} \cdot p_{B}(x) p_{X}(x) d x}{\int p_{B}(x) p_{X}(x) d x} \\
& =\frac{\int_{|x| \geq 1+\varepsilon} x^{2} \cdot p_{B}(x) p_{X}(x) d x}{\int_{|x| \geq 1+\varepsilon} p_{B}(x) p_{X}(x) d x} \geq(1+\varepsilon)^{2}>1 .
\end{aligned}
$$

Therefore, $\psi_{\text {MMSE }}$ is expansive. Since it is also increasing, the associated $\phi_{\text {MMSE }}$ is $C^{\infty}$ (Theorem II.2) hence it is proper and continuous. As a result of [2, Proposition 2.4], since $\psi_{\mathrm{MMSE}}=\operatorname{prox}_{\phi_{\mathrm{MMSE}}}$, the penalty $\phi_{\mathrm{MMSE}}$ cannot be convex. Similar examples can be built in higher dimensions.

\section{REFERENCES}

[1] T. Blu and F. Luisier. The SURE-LET approach to image denoising. IEEE Trans. Image Proc., 16(11):2778-2786, 2007.

[2] P. L. Combettes and J.-C. Pesquet. Proximal thresholding algorithm for minimization over orthonormal bases. SIAM J. Optim., 18:1351-1376, 2007.

[3] D. Donoho. Denoising by soft-thresholding. IEEE Trans. Inform. Theory, 41(3):613-627, May 1995.

[4] D. L. Donoho. Compressed sensing. IEEE Trans. Inform. Theory, 52(4):1289-1306, 2006.

[5] S. Kay. Fundamentals of Statistical Signal Processing : Estimation Theory. Signal Processing. Prentice Hall, 1993.

[6] P. Moulin and J. Liu. Analysis of multiresolution image denoising schemes using generalized Gaussian and complexity priors. IEEE Trans. on Information Theory, 45(3):909-919, April 1999.

[7] M. Nikolova. Model distortions in Bayesian MAP reconstruction. Inverse Problems and Imaging, 1(2):399-422, 2007. 
[8] M. Raphan and E. P. Simoncelli. Learning to be Bayesian without supervision. In B. Schölkopf, J. Platt, and T. Hofmann, editors, Adv. Neural Information Processing Systems (NIPS*06), volume 19, pages 1170-1177, Cambridge, MA, may 2007. MIT Press.

[9] C. Stein. Estimation of the mean of a multivariate normal distribution. Ann. Statist., 9(6):1135-1151, 1981.

[10] J. S. Turek, I. Yavneh, M. Protter, and M. Elad. On MMSE and MAP denoising under sparse representation modeling over a unitary dictionary. submitted to Applied and Computational Harmonic Analysis, Technion University, 2010.

[11] H. Zayyani, M. Babaie-Zadeh, and C. Jutten. Bayesian pursuit algorithm for sparse representation. In Proceedings of ICASSP2009, pages 1549-1552, Taipei, Taiwan, 19-24 April 2009. 